\title{
Représenter volailles et volaillères dans la peinture italienne du Cinquecento
}

Analogies physiques et associations alimentaires dans les tableaux de Campi et Passerotti

Represent poultry and sellers of poultry in the 16th Century Italian painting. Physical analogies and food associations in Campi and Passerotti's pictures

\section{Valérie Boudier}

\section{OpenEdition \\ Journals}

Édition électronique

URL : http://journals.openedition.org/ethnoecologie/3294

DOI : 10.4000/ethnoecologie.3294

ISSN : 2267-2419

Éditeur

Laboratoire Eco-anthropologie et Ethnobiologie

Référence électronique

Valérie Boudier, «Représenter volailles et volaillères dans la peinture italienne du Cinquecento », Revue d'ethnoécologie [En ligne], 12 | 2017, mis en ligne le 18 décembre 2017, consulté le 19 avril 2019. URL http://journals.openedition.org/ethnoecologie/3294 ; DOI : 10.4000/ethnoecologie.3294

Ce document a été généré automatiquement le 19 avril 2019

\section{c)}

Revue d'ethnoécologie est mis à disposition selon les termes de la licence Creative Commons Attribution - Pas d'Utilisation Commerciale - Pas de Modification 4.0 International. 


\section{Représenter volailles et volaillères dans la peinture italienne du Cinquecento}

Analogies physiques et associations alimentaires dans les tableaux de Campi et Passerotti

Represent poultry and sellers of poultry in the 16th Century Italian painting. Physical analogies and food associations in Campi and Passerotti's pictures

\section{Valérie Boudier}

1 Attesté dans les sources historiques, présent en littératures et en images - illustratives ou artistiques -, le thème de la volaillère ${ }^{1}$, et donc de la vente de volailles, documente l'usage d'objets quotidiens paysans, témoigne de pratiques d'élevage et d'abattage, participe enfin d'une histoire de l'alimentation. À la croisée de disciplines, ce thème a été exploré, notamment par l'histoire économique, culturelle et sociale mais aussi par l'ethnoanthropologie. Concernant les images, elles sont objets légitimes d'étude en tant que sources visuelles servant de support à la reconstitution de l'histoire et de la culture alimentaires. Cette perspective très féconde est toutefois loin d'épuiser la compréhension et la connaissance des images, car elle ne prend pas en considération les enjeux artistiques de la représentation, ni la logique figurative qui lui est propre. Or, si les images doivent être exploitées en anthropologie historique, ce n'est pas seulement en tant que documents visuels permettant de compenser certaines lacunes des sources textuelles; elles doivent être étudiées comme représentations figuratives en tant que telles.

2 Les œuvres peintes ne sont nullement aisées à déchiffrer et les scènes de vente de volailles proposées par Vincenzo Campi (1536-1591) et Bartolomeo Passerotti (1529-1592) ne sont pas, évidemment, des instantanés de la vie populaire du xvI ${ }^{\mathrm{e}}$ siècle italien. Cuuvres complexes, ces peintures de genre (Boudier 2010) nécessitent une interprétation croisant leur analyse plastique avec l'étude de textes contemporains de tous types (littéraire, 
artistique, culinaire), et cela pour permettre une ouverture de l'analyse vers un arrièreplan social et culturel.

3 Ces deux artistes, le Crémonais Vincenzo Campi et le Bolonais Bartolomeo Passerotti, font naître, en Italie, à la fin du Cinquecento, des scènes peintes d'un genre ${ }^{2}$ nouveau: des compositions aux dimensions imposantes où évoluent poissonniers, cuisiniers et volaillères parmi une multitude de produits alimentaires. Les volatiles et les vendeuses ${ }^{3}$ qui les accompagnent possèdent, chez ces deux artistes, une place et un traitement tout à fait originaux. Ils semblent incarner les modalités de regard du spectateur dans l'économie des tableaux de genre des deux artistes italiens.

\section{Vincenzo Campi}

4 Vincenzo Campi appartient à une famille d'artistes. Petit-fils, fils et frère de peintres, il fonde son propre atelier en 1573. Quelques années plus tard, vers 1575-1576, le peintre propose, outre des œuvres religieuses, des tableaux figurant des sujets ayant trait à l'aliment. Dans l'atelier, au moment du décès du peintre, en 1591, se trouvent encore six tableaux essentiels pour notre propos. Mis à part les Mangeurs de ricotta ${ }^{4}$, vendus entretemps, cinq tableaux se retrouvent dans le testament de la veuve du peintre daté de 1611 : une Fruitière ${ }^{5}$, des Poissonniers ${ }^{6}$, des Volaillers (Figure 1), un Déménagement ${ }^{7}$ et une Cuisine ${ }^{8}$. Autant d'œuvres servant de modèles ${ }^{9}$ à l'exécution d'autres réalisations picturales. De fait, une des spécificités de la production de ce peintre est de travailler la série à partir d'un fonds de tableaux qu'il garde dans son atelier. Hormis le Déménagement, toutes ces toiles appartiennent aujourd'hui au fonds de la Pinacothèque de Brera, à Milan. Une seconde série connue de l'artiste est conservée, aujourd'hui encore, au château de Kirchheim en Bavière et décorait la salle à manger de Hans III Fugger (1531-1598). Elle est constituée de cinq tableaux: une Fruitière ${ }^{10}$, des Volaillers (Figure 2), une Poissonnière ${ }^{11}$ et deux versions de Poissonniers ${ }^{12}$; ces œuvres, pour certaines, sont quasi identiques aux originaux du musée milanais. 
Figure 1 : Vincenzo Campi, Volaillers, vers 1580, huile sur toile, 145 x $215 \mathrm{~cm}$, Milan, Pinacoteca di Brera

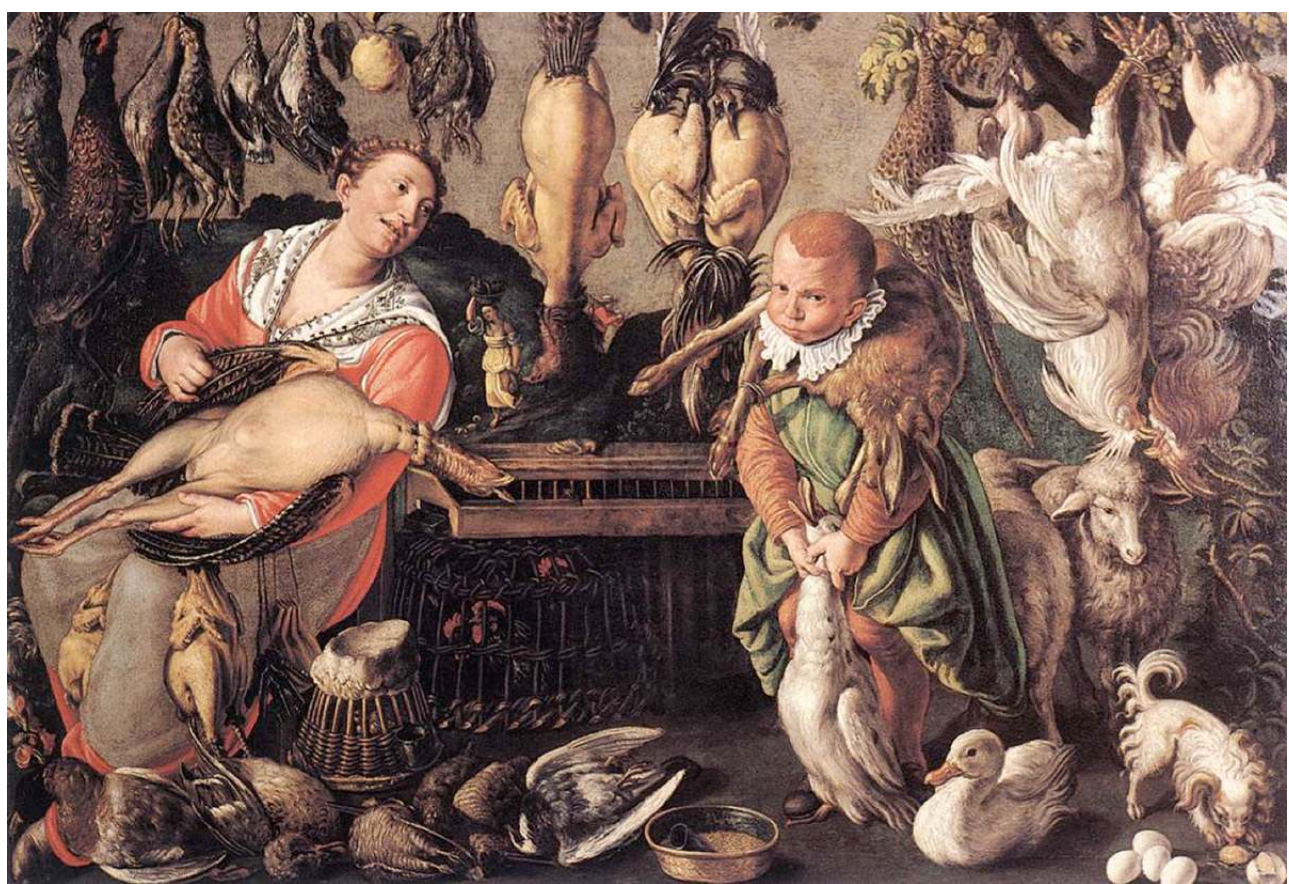

Figure 2 : Vincenzo Campi, Volaillers, vers 1580, huile sur toile, 135 x $220 \mathrm{~cm}$, Kirchheim, coll. Fugger

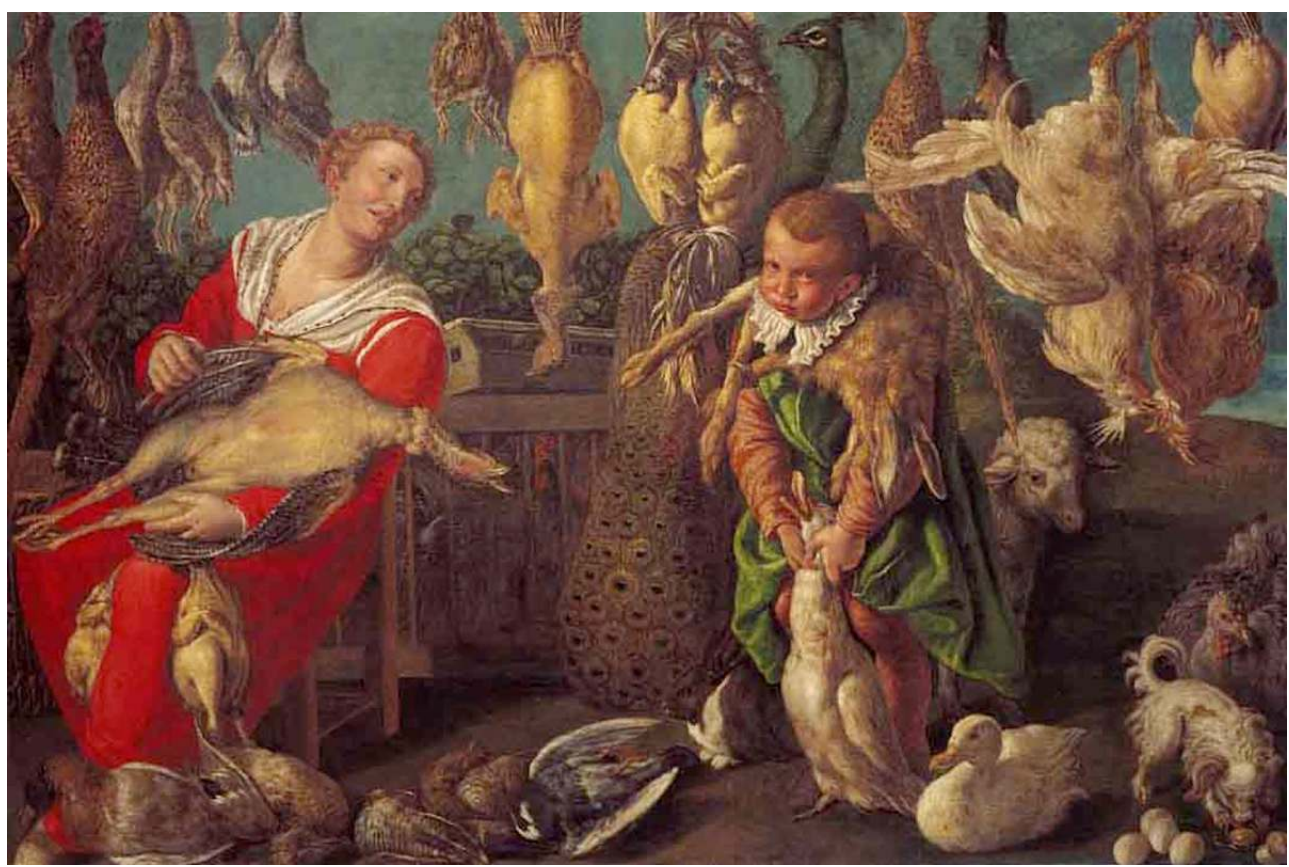

5 Selon certains historiens de l'art (De Logu 1962, Wind 1975), les quatre tableaux de la Pinacothèque de Brera s'inscriraient dans un contexte particulier: le goût à l'époque pour les peintures allégoriques des Quatre saisons et pour celles des Quatre éléments. Dans cette optique, d'une part, la Fruitière serait associée à l'Automne, la Cuisine à l'Été, les Poissonniers à l'Hiver et les Volaillers au Printemps. D'autre part, la Fruitière s'apparenterait à la Terre, la Cuisine au Feu, les Poissonniers à l'Eau et les Volaillers à l'Air. Par cette approche, 
les toiles de Vincenzo Campi seraient à rapprocher de celles de Giuseppe Arcimboldo (1527-1593), artiste réputé pour ce type de compositions allégoriques. Ainsi, pour figurer l'allégorie de l'Air (Figure 3), le peintre milanais assemble, regroupe et juxtapose un même genre d'êtres sensibles: des oiseaux, des plus petits aux plus grands, des plus courants aux plus rares. Mais cette lecture rapide des œuvres de Campi doit être nuancée car ce ne sont pas quatre mais cinq tableaux qui restaient au sein de l'atelier. Les tableaux du Crémonais sont complexes : ils se répondent par leur thème de représentation, sont à lire comme des jeux visuels et proposent des sous-entendus sexuels par le biais de gestes équivoques, d'analogies physiques et d'associations alimentaires.

Figure 3 : Guiseppe Arcimboldo, Air, vers 1566, huile sur toile, 74 x 56 cm, Bâle, coll. privée

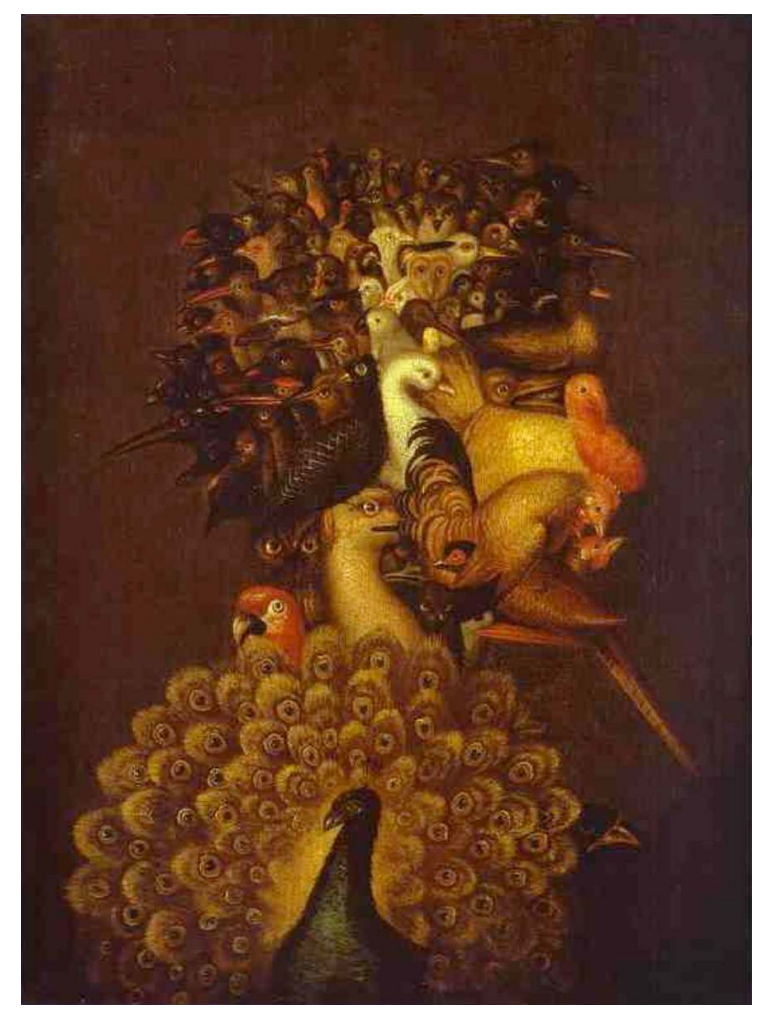

Vincenzo Campi conçoit systématiquement ses œuvres pour qu'elles soient exposées ensemble. Il travaille les principes de compositio, de varietas et de copia ${ }^{13}$ tout en proposant des jeux visuels, basés sur la reprise de motifs d'une composition à l'autre. À la fois pratique d'atelier et principe créateur, l'assemblage de motifs structure le tableau intitulé la Poissonnière (Figure 4) de Kirchheim et permet un jeu visuel entre cette œuvre et l'une des deux versions des Poissonniers (Figure 5), conservées au même endroit. Campi représente sa Poissonnière suivant les caprices de son imagination plastique. C'est une scène d'extérieur où, dans la partie inférieure, s'exposent des plats, bassines et paniers contenant des poissons. Le plat en cuivre avec une truite en bas à gauche de la Poissonnière se retrouve à l'identique dans la partie centrale du tableau des Poissonniers. Le baquet rempli d'anguilles, le panier plein d'écrevisses et le billot supportant une hydre coupée en deux et sanguinolente sont identiques mais disposés différemment dans l'une et l'autre représentations. 
Figure 4 : Vincenzo Campi, Poissonnière, vers 1580, huile sur toile, $145 \times 215 \mathrm{~cm}$, Kirchheim, coll. Fugger

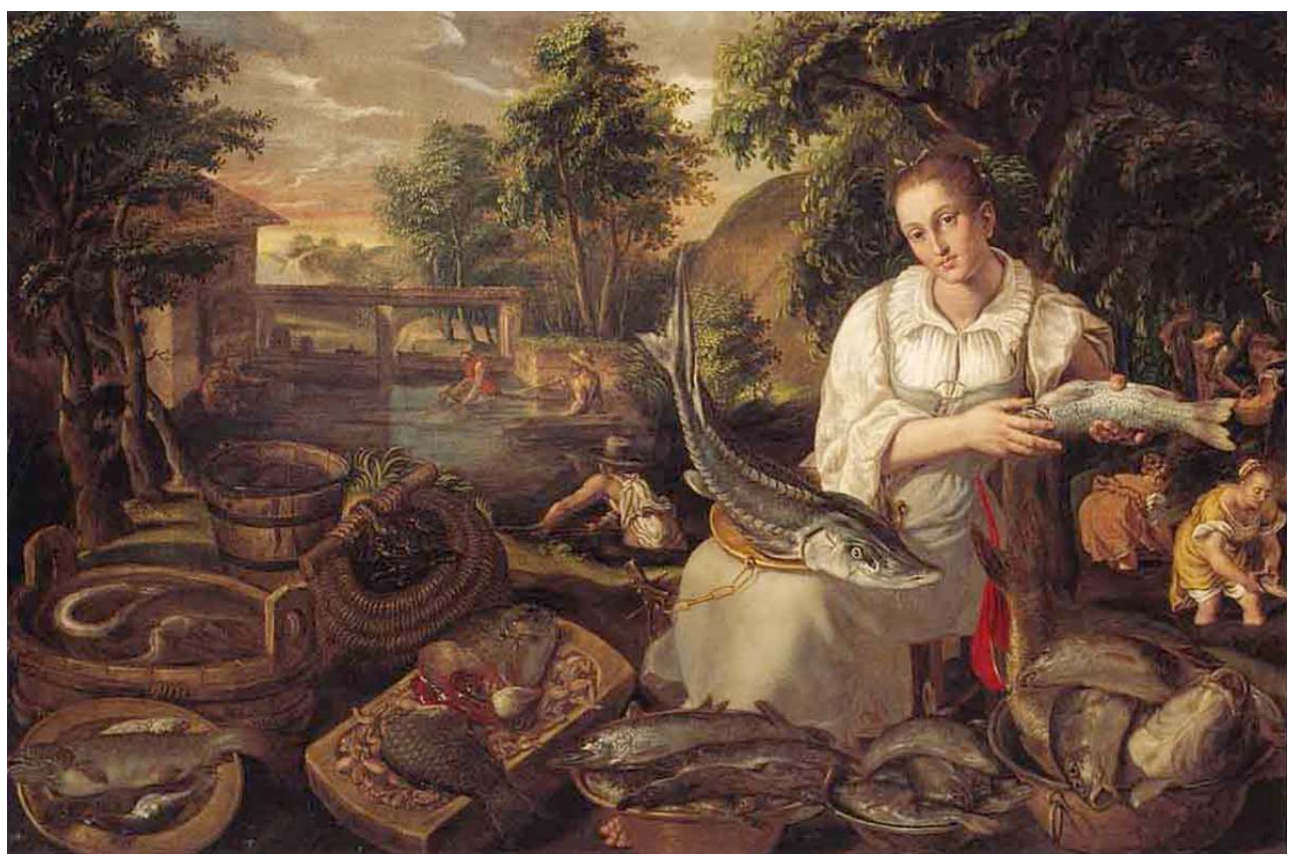

Figure 5 : Vincenzo Campi, Poissonniers, vers 1580, huile sur toile, $145 \times 215 \mathrm{~cm}$, Kirchheim, coll. Fugger

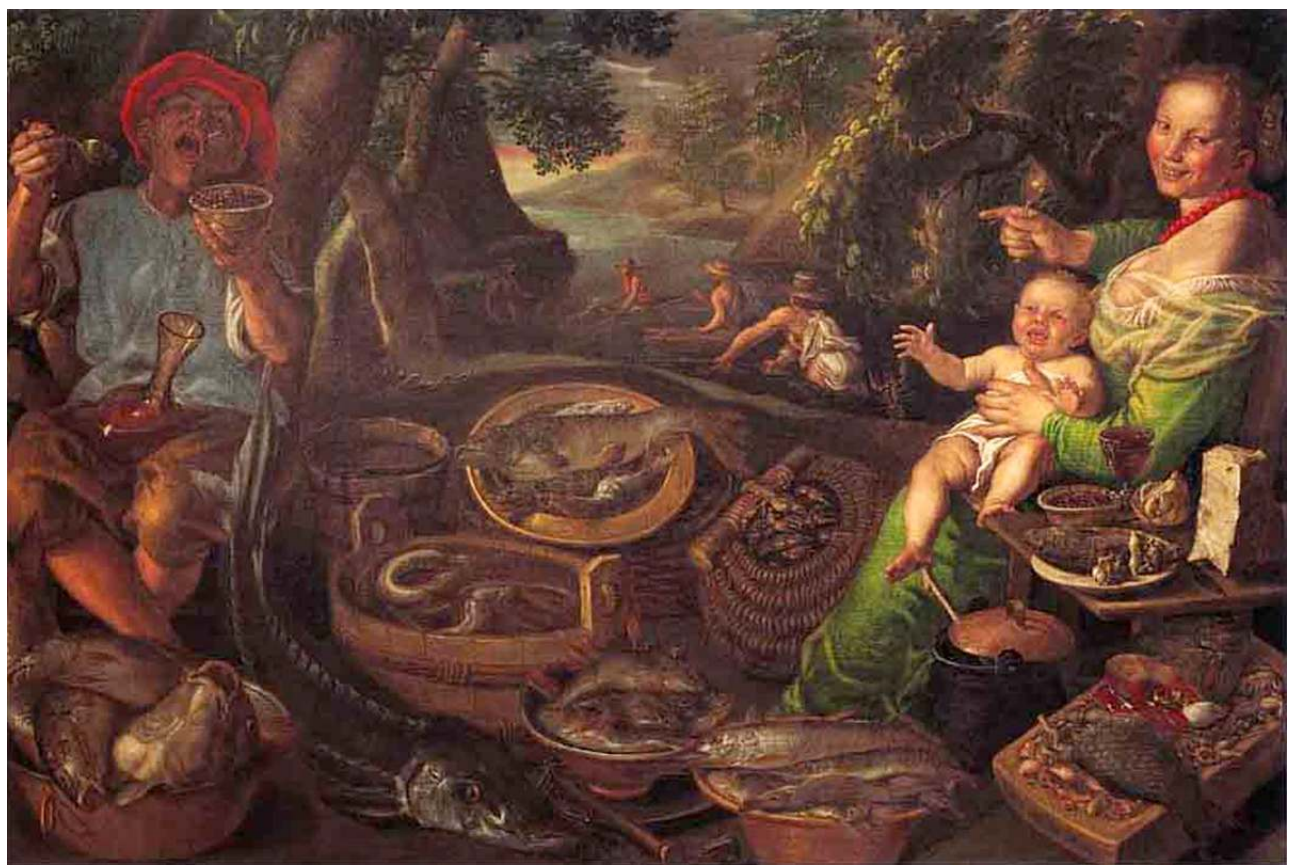

Dans les Volaillers (Figure 1), Campi applique les mêmes principes que ceux régissant les précédents tableaux : une compositio reposant sur l'idée d'assemblage, une copia réglée par la varietas. Il développe également l'utilisation d'une poétique du multiple et des contraires. Il y a déjà opposition de l'état. Ainsi, au sein de la composition, une étonnante opposition entre animaux morts et animaux vivants est mise en place : les poules en cage et les deux canards du premier plan sont en vie ; les autres volatiles sont présentés morts. 
À gauche, canards colverts, poulets, bécasses, dinde, faisans, pendus ou déposés à terre, s'opposent aux deux moutons et au chien debout sur leurs pattes. Ce contraste entre animaux morts et vifs est renforcé par celui créé entre volatiles plumés ou non. À droite, l'extravagant plumage blanc des deux coqs pendus par les pattes s'oppose à la paire de poulets plumés et bien gras - peut-être des chapons - quasiment au centre du tableau. Les deux volailles accrochées ensemble par le bec et tombant de chaque côté de la jambe de la femme tranchent par la nudité de leur carcasse avec l'ensemble des oiseaux morts emplumés du premier plan de la représentation. Le peintre joue encore sur les contraires lorsqu'il mélange animaux domestiques et animaux sauvages ${ }^{14}$ et lorsqu'il associe des volatiles femelles et mâles: poules et coqs, canards colverts des deux sexes, faisan et faisane.

L'élevage des animaux de basse-cour est une activité de femmes et d'enfants en Italie au Cinquecento (Patrone 1989). Dans l'image, la figure féminine avec sa dinde rappelle aussi l'activité de plumage et de vente allouée traditionnellement aux femmes. À ses côtés, son pendant masculin, le garçon, est en train de gaver le canard qu'il a immobilisé entre ses jambes. Il porte autour du cou, non pas un lapin pourtant considéré comme un animal de basse-cour, mais un lièvre, son pendant sauvage. À l'instar de ce qu'il propose dans sa Poissonnière et ses Poissonniers, le peintre s'attache à rendre, dans les Volaillers, la variété et l'abondance des espèces animales.

9 Cette lecture des tableaux de Campi comme des mises en scène de la diversité et des jeux visuels basés sur le multiple et le contraire, peut être confortée par une analyse de l'environnement culturel dans lequel évoluait l'artiste. De fait, autour des années 1573-1575, Vincenzo Campi travaillait pour la sphère influente du cardinal Carlo Borromeo, à Milan. Or, dix ans auparavant, l'humaniste et homme de lettres milanais Gerolamo Cardano (1501-1576) comptait parmi les protégés du cardinal. Cardano se fixe à Bologne en 1562 et part pour Rome autour de 1570. Le peintre n'a donc pas pu le croiser à Milan. Mais si les hommes voyagent, les écrits restent. Dans les années 1570, deux des ouvrages de Gerolamo Cardano circulaient dans la sphère intellectuelle et religieuse du cardinal milanais : le De subtilitate de $1550^{15}$ et le De rerum varietate de $1557^{16}$. Ouvrages à succès, le premier comporte vingt et un livres et s'apparente à une encyclopédie universelle des sciences naturelles et des inventions; le second compte dix-sept livres et reprend, en les complétant, des descriptions et analyses du premier. L'auteur y relie « un principe unique, indivisible et absolument simple» (Baldi \& Canziani 2002: 13) à la variété et à l'abondance des choses humaines et divines, des phénomènes naturels, des genres et des espèces qui peuplent la mer, le ciel et la terre.

Devant le double processus du passage de l'un au multiple et du multiple à l'un, autrement dit, la diffusion du principe divin dans la multiplicité des êtres sensibles, Gerolamo Cardano s'émerveille. Dans le De rerum varietate, l'auteur expose sa fascination pour les secrets de la nature et pour l'ordre divin, pour la variété de la nature et la présence divine dans chaque être sensible. «Par la variété, la nature est belle » (Baldi \& Canziani 2002 : 14) écrit le penseur pour qui la varietas est présente par et dans la multiplicité des êtres sensibles. Alors que la notion d'espèce est essentielle chez Cardano parce qu'elle fonde l'ordre des différences et des similitudes, les animaux rassemblés sur les toiles de Vincenzo Campi appartiennent à une même espèce, à un même genre d'êtres sensibles. Les représentations peintes de l'artiste crémonais semblent s'inscrire dans la même ligne de pensée que Gerolamo Cardano. Cependant, à cette lecture docte peut être associée une autre lecture plus légère. 
11 Les tableaux de Campi, nous l'avons vu, se font écho par leur thème de représentation ${ }^{17}$ et leur conception formelle ${ }^{18}$. Le peintre opère des reprises d'une version à l'autre des Volaillers puisque la première sert de modèle à la seconde. Les Volaillers (Figure 1) de la Pinacothèque de Brera proposent un contexte campagnard qui n'est pas innocent ${ }^{19}$, où deux personnages sont mis en scène : une marchande - portant tablier, clés et bourses et un garçon volailler; chacun exhibe à sa manière un volatile. La femme présente une dinde, en partie plumée, ailes déployées, et l'adolescent gave un canard tout en regardant le spectateur. Dans le dessein de le faire sourire, Vincenzo Campi recourt ici à des analogies visuelles et joue avec les formes plastiques. Il rapproche visuellement la peau de la vendeuse de celle de sa dinde tandis qu'il figure le jeune adolescent avec la bouche pleine alors qu'il gave lui-même un canard ${ }^{20}$. Il exerce du haut vers le bas un geste de resserrement du cou du volatile qu'il tient entre ses cuisses. Le bec entrouvert du canard, orienté vers le haut, prend place à la hauteur du sexe du garçon. L'idée d'érection n'est pas improbable lorsque l'on sait que Vincenzo Campi semble proposer des détails plastiques équivoques dans bon nombre de ses peintures. Il répond en cela au goût très prononcé de ses contemporains pour l'équivoque, qu'elle soit plastique ou littéraire. De fait, dans l'Italie du Cinquecento, une littérature de l'équivoque est très en vogue, celle d'auteurs tels que Francesco Berni, Matteo Bandello ou Il Lasca. Cette littérature qui se caractérise par un recours constant à des doubles sens obscènes semble avoir intéressé le peintre. À première vue, les Volaillers ne contreviennent en rien à la bienséance. Dans l'image, il suffit d'un rien, d'une main glissée le long d'une cuisse animale, de joues gonflées, d'un adolescent gavant un canard pour que le sens caché se révèle au regard du spectateur.

Dans la seconde version des Volaillers (Figure 2), conservée au château de Kirchheim, on distingue, autour du garçon, un lapin, un paon, un dindon, les deux mêmes canards que précédemment, le petit chien lapant un jaune d'œuf, tous vivants. Deux coqs plumés pendus et morts renvoient aux deux chapons ou poulets déplumés, quasiment au centre de la composition tandis que la poule faisane fait écho au faisan placé près de la vendeuse. Cette dernière est entourée, dans la partie haute de l'image, du faisan, de deux perdrix grises, de grives et, dans la partie basse, de deux canards, trois bécasses et un pigeon. Le geste du garçon volailler n'est plus aussi clair que précédemment car la bassine de cuivre remplie de grains de maïs a été supprimée. La majorité des historiens de l'art ${ }^{21} \mathrm{y}$ voit un geste de strangulation alors que c'est celui d'un glissement le long d'une forme oblongue qu'il faut lire. 
Figure 6 : Bartolomeo Passerotti, les Vendeuses de poulets et de légumes avec un enfant, vers 1580 , $113 \times 95 \mathrm{~cm}$, Berlin, Gemäldegalerie

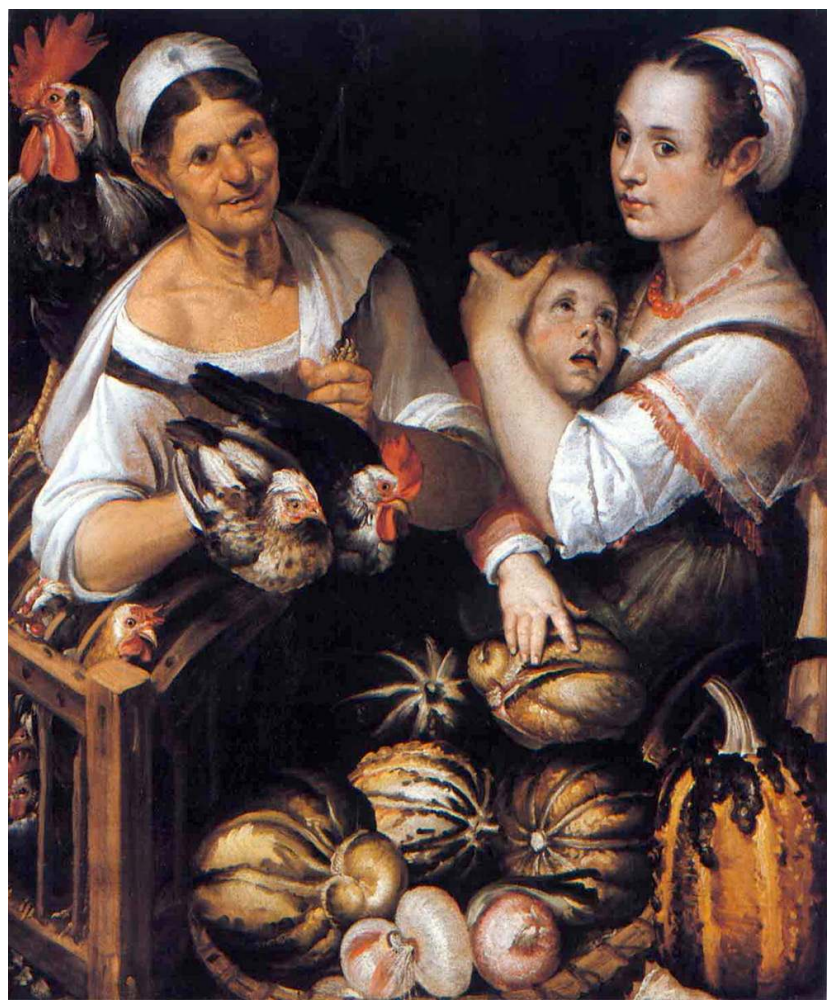

13 La volaillère tient dans ses bras la même dinde morte que dans la peinture précédente, comme une mère tient un enfant endormi dans ses bras. L'idée d'une relation intime entre la marchande de volailles et sa dinde est reprise dans un autre tableau, celui de Bartolomeo Passerotti intitulé Vendeuses de poulets et de légumes avec un enfant (Figure 6), où une jeune femme protège - avec fermeté - un enfant de son bras gauche. Elle semble être l'incarnation même de la poule qui protège et nourrit ses poussins au sein du poulailler. La mère poule est une figure déjà présente dans les traités d'économie domestique de l'époque où il est question de l'élevage des gallinacés. Dans son traité intitulé Le Vinti giornate dell'agricoltura et de'piaceri della villa de 1569, Agostino Gallo atteste bien que « les poussins [...] qu'on mettra avec la dite poule, sous quelques paniers bien chaudement par cinq jours, ou six, sans qu'ils aient faute de mangeaille, ni de boire, tant qu'ils sont sous la garde de leur mère » (Gallo 1571 : 213). Dans le tableau, la jeune mère à l'enfant est associée à une vieille volaillère qui semble vanter la qualité d'un petit coq et d'une poule qu'elle tient des deux mains. Son châle a glissé de son épaule droite, dévoilant une gorge ridée et un cou plissé. Si Passerotti associe la jeune femme à un enfant, il rapproche visuellement et symboliquement la vieille au coq situé à l'arrièreplan. Cette association prête à sourire lorsque l'on sait que le coq est réputé «vil et couard [...] \& surtout qu'il aime les poules, \& soit hardi, \& courageux, qu'il s'attaque à toute bête pour la défense de ces amoureuses » (Gallo 1571 : 231). À l'instar de Vincenzo Campi, Bartolomeo Passerotti utilise un ensemble d'artifices visuels et d'associations de lieux communs pour provoquer le rire chez le spectateur. 


\section{Bartolomeo Passerotti} quatre œuvres. Aucun document n'atteste l'existence du cycle avant son achat, en 1603, par le marquis Ciriaco Mattei di Calcara alors que ces quatre tableaux furent vraisemblablement exécutés autour de 1580 (Testa 1995). L'artiste qui les signe, Bartolomeo Passerotti, est alors à la tête d'un atelier florissant à Bologne mais est aussi connu à Rome pour avoir travaillé pour la cour pontificale de Pie V et celle de Grégoire XIII. Il est peu étonnant ainsi que ces tableaux qui appartiennent à la catégorie des « composizioni dell' allegrezze e de' risi » (Lomazzo 1971), définie en 1584, ornent la salle de réception d'un dignitaire romain, proche de Filippo Neri, au sein, non pas de son palais de la rue des Botteghe Oscure au centre de Rome, mais dans sa villa suburbaine della Navicella, sur le Celio, à la campagne.

Figure 7 : Bartolomeo Passerotti, Volaillères, vers 1580, huile sur toile, 114 x 152,5 cm, Florence, coll. Longhi

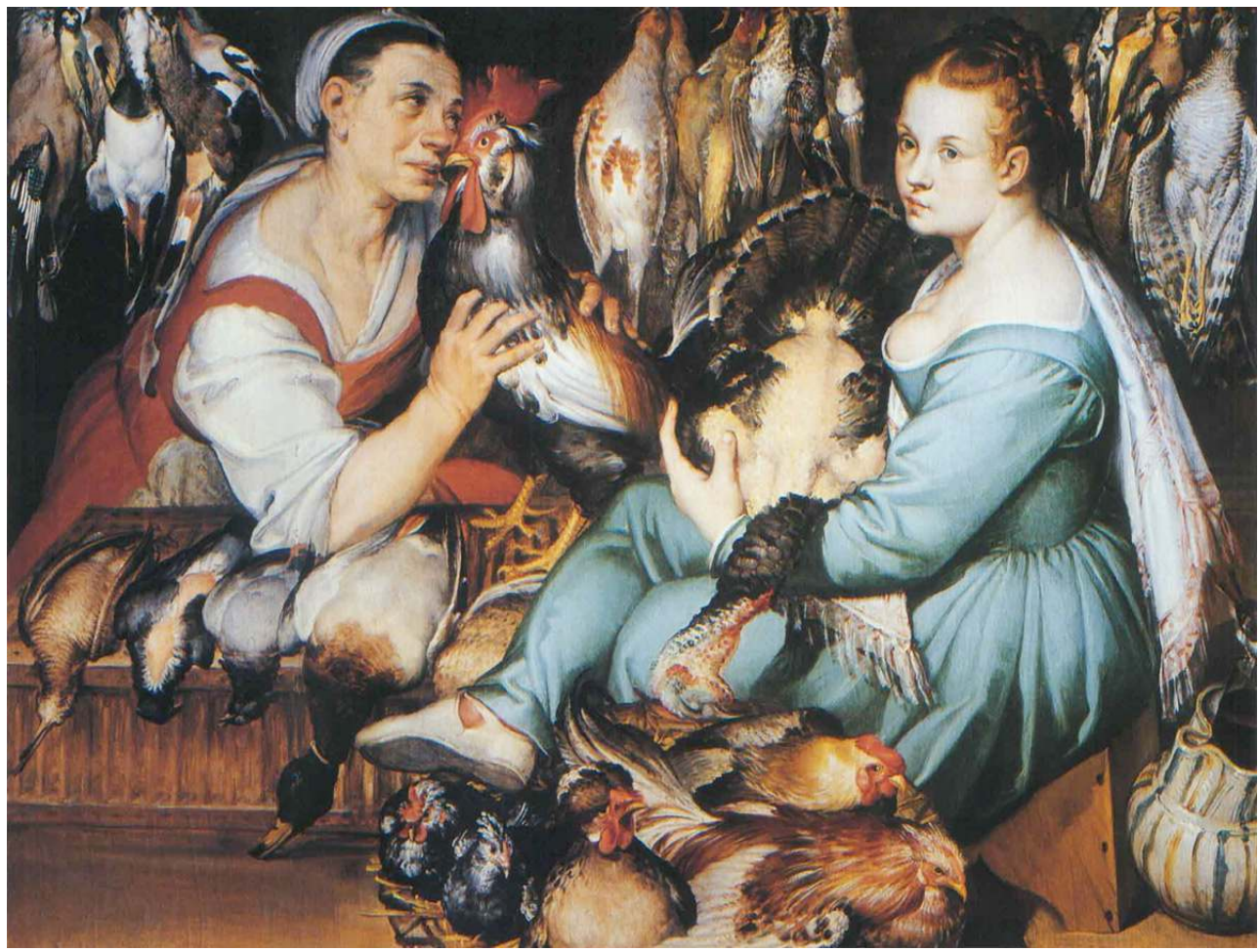
quelconque sens caché dans ces tableaux (Testa 1995). L'inventaire décrit le cycle de façon assez sommaire, regroupant toutes les denrées et additionnant l'ensemble des personnages représentés sur les toiles: «quatre tableaux de Passerotti où sont représentés de la viande taillée en morceaux et des poissons, des oiseaux, des tortues et des homards avec des figures d'hommes, et de femmes c'est-à-dire deux par tableau et dans un, on voit un enfant $»^{22}$.

Le texte de l'inventaire met en avant l'importance des aliments. Ces derniers structurent fortement les images et mettent en scène les personnages. L'ensemble des quatre peintures est homogène par sa cohérence plastique et thématique. Dans chaque toile, sur 
une profondeur réduite, des denrées carnées - poissons ou viandes - encadrent des personnages couplés. Un homme et une femme pour les Poissonniers, deux hommes pour les Bouchers, deux femmes pour les Volaillères et deux hommes et un enfant pour les Poissonniers. Cet ensemble, que j'appelle le cycle Mattei, est à envisager dans sa globalité. Conçus pour être exposés ensemble, les quatre tableaux peuvent être interprétés indépendamment mais se répondent aussi entre eux. Souvent, la clé interprétative d'une peinture en particulier est contenue dans un autre tableau du même cycle.

Chaque peinture présente, en apparence, une scène de genre mais propose en même temps une autre lecture, voire plusieurs autres lectures, qui s'ajoutent à la première. Par ce processus, ces tableaux s'apparentent à des allégories picturales. Si l'allégorie rhétorique est une figure qui consiste à dire une chose pour en faire comprendre une autre, dans l'allégorie picturale, le fonctionnement est inverse. L'allégorie rhétorique va de l'abstrait au concret, du mot à l'image ; l'allégorie picturale fait concevoir l'abstraction à partir de l'image. Dans le premier cas, l'allégorie fait image pour éclairer le sens, dans le second cas, c'est l'image qui fait sens. Pour donner un sens allégorique à ses scènes de vente alimentaire, Passerotti utilise systématiquement un ensemble d'artifices visuels et des associations de lieux communs parfois obscènes, souvent risibles.

Dans la première version des Poissonniers, par exemple, la moule géante et béante associée à la vieille femme évoque un sexe féminin. Cette moule démesurée est mise en présence d'une autre moule de forme phallique se dressant devant le vieil homme. L'âge avancé des deux protagonistes accentue l'aspect comique de la connotation sexuelle. Cet aspect risible est renforcé par l'analogie physique existant entre le poisson-globe tenu par la vieille femme et le visage de cette dernière : même rotondité de la tête, mêmes incisives inférieures et mêmes petits yeux vifs. Le gonflement général du poisson et sa bouche ouverte évoquent le vide de la tête féminine et le vent des paroles qu'elle déverse sur son partenaire.

Dans l'autre version des Poissonniers, une nette ressemblance associe le poissonnier hurlant et les brochets qu'il propose à la vente: les mâchoires et les regards sont similaires. Ces analogies physiques sont à mettre en lien avec les théories de la physiognomonie très répandues à l'époque. Dans son Sculptura de 1504, Pomponius Gauricus reprend l'interprétation des fondateurs antiques de cette science et explique de façon systématique que "les ressemblances physiques d'un individu avec une espèce animale quelconque passent pour prouver qu'il en possède aussi les caractères biologiques et psychiques» (Gauricus 1969: 116). Dans les tableaux de Passerotti, les visages humains aux traits déformés par des grimaces rapprochent l'homme de l'animal. Mais les poissons, mammifères et oiseaux sont traités aussi comme les reflets ou les transpositions de comportements humains ; ils deviennent animaux humanisés.

L'aspect anthropomorphe des animaux et l'animalité de l'homme relèvent de l'hybridation et du mélange des règnes, un procédé d'une remarquable efficacité que l'on retrouve dans le tableau intitulé les Bouchers. À l'instar des tableaux précédents, c'est le rapprochement physique de l'homme avec l'animal qui transforme l'homme en bête. En exhibant son attribut, la hure de sanglier, le jeune boucher devient porteur de la puissance sexuelle du porc sauvage réputé comme l'un des animaux les plus luxurieux depuis l'Historia animalium d'Aristote.

21 Quant au tableau intitulé Les Volaillères (Figure 7), il présente deux femmes entourées d'oiseaux sauvages morts, dans le registre supérieur de l'image, de volatiles sauvages pouvant être élevés en captivité, dans le registre médian et de volailles domestiques 
vivantes dans le registre inférieur. Les deux femmes posent en touchant ou présentant un volatile. La vieille étreint un coq et la jeune exhibe une dinde tout en prenant à parti le spectateur par son regard insistant. À chaque personnage son signe distinctif : un coq ici, une dinde là, qui sont autant de symboles évoquant, par leur forme ou leur nature, autre chose que ce qui est peint, et cela en vertu du principe de correspondances analogiques si présent au XVI ${ }^{\mathrm{e}}$ siècle. Ici, l'analogie fonctionne sur deux registres différents : dans le cas de la jeune femme associée à la dinde, l'analogie est formelle, dans celui de la vieille femme et de son signe distinctif, le coq, l'analogie est intrinsèque ou essentielle.

L'absence de caroncule extensible nous montre que le volatile tenu par la jeune femme est incontestablement une femelle. La dinde est présentée de façon ridicule : le croupion de l'oiseau est juste sous le nez de la vendeuse. Les peaux de l'animal et de la jeune femme s'apparentent étrangement. Grasses et bien roses toutes les deux, la dinde et la femme appellent au péché de chère et de chair, thème récurrent $a u \mathrm{XVI}^{\mathrm{e}}$ siècle en peinture comme en littérature. Nous songeons ici à la nouvelle, issue de I Marmi de 1553, du polygraphe et poète italien Anton Francesco Doni, dans laquelle le narrateur tombe amoureux d'une "honnête" femme lors d'un séjour estival sur ses terres: "J'allais guignant çà et là, comme un oisif insouciant ; j'attrapais les oiseaux à la pipée, j'allais à la chasse et autres passe-temps, et lorsque cela me réussissait, j'allais conter fleurette. En un rien de temps, je trouvai ce que je cherchais. Il arriva en effet de la ville une fraîche petite femme, mariée depuis quelques mois seulement, un petit salé, je puis vous l'assurer, qui vous aurait fondu dans la bouche " (Doni 1993 : 1136). Le texte de Doni a d'autant plus d'intérêt qu'il met l'accent sur une autre problématique qui travaille le tableau de Passerotti : les liens du monde rural avec le monde citadin.

Dans l'image, l'association formelle ne s'arrête pas là. La jeune femme s'expose et semble vanter ses charmes car elle est placée devant l'étal - voire sur l'étal : visuellement, son tabouret forme la continuité de la cage. Elle devient marchandise parmi les produits vendus. Tandis qu'elle croise les jambes, la jeune volaillère lève sa jambe droite et laisse voir sa chaussure et son bas. Cette «jambe en l'air $»^{23}$ est un des signes de l'exhibitionnisme féminin (Bougoux 1992); ce motif obscène est repris ici par Passerotti. À cette idée, le peintre associe celle développée par certains auteurs de l'époque ${ }^{24}$ selon laquelle les activités de vente sont, pour les femmes, une couverture servant à masquer leur activité de prostitution. Si la jeune femme vend ses charmes, sa vieille compagne est alors l'entremetteuse qui sert d'intermédiaire entre le client et la prostituée. Les attraits physiques de la jeune femme rappellent d'ailleurs étrangement les vertus énoncées par Baldassare Pisanelli dans son Trattato della natura de cibi et del bere de 1584, concernant la dinde qui, si elle doit être mangée « jeune, \& engraissée à la campagne tuée d'hiver, \& mortifiée à l'air, [...] donne abondant \& très bon nourrissement, restaure les débiles, \& convalescents, accroît la semence, \& incite Vénus » (Pisanelli 1620 : 115).

L'analogie formelle entre la jeune femme et la dinde semble donc renforcée par la parenté des vertus de l'animal et de la femme. Mais l'analogie formelle joue sur un autre registre car, au-delà des deux personnages féminins associés dans le tableau, chaque personnage forme un couple avec son animal : la jeune femme avec sa dinde, la vieille femme avec son $\operatorname{coq}^{25}$. Concernant ce dernier couple, nous avons affaire à un « véritable » couple comme l'atteste la langue que la vieille tend vers le bec du coq. Cette idée est renforcée par la présence de duos d'oiseaux, un peu partout sur la toile, disposés en couple avec un mâle et une femelle. Dans la partie supérieure, deux vanneaux huppés sont associés à deux perdrix grises et deux gros-becs casse-noyaux. Dans la partie médiane, deux pigeons 
bisets côtoient deux colverts. Enfin, dans le registre inférieur, contrastant avec ces volatiles morts, des volailles domestiques vivantes sont présentées: un poulet et une poulette nains, une poularde, un chapon et un coq de Padoue.

À ces couples répond celui formé par la vieille qui sert et embrasse son coq. Or, le coq fait partie des animaux réputés particulièrement libidineux par la précocité de l'apparition de ses testicules, sa puissance sexuelle et sa forte production de semence lui permettant de féconder plus de dix poules au sein du poulailler. «À chacune douzaine de poules, c'est assez d'un bon coq" écrit Charles Estienne dans son Agriculture, et maison rustique (Estienne \& Liebault 1671:67). Avec le motif de la vieille et son coq, Passerotti exploite un thème déjà souvent traité par les artistes flamands: le couple du vieux fermier et de sa poule. Citons les gravures de Harmen Jansz Müller (Figure 8) ou de Hans Liefrink illustrant le thème du Vieux paysan avec sa poule (Figure 9), où les artistes insistent sur l'attirance sexuelle de l'homme pour l'animal. Dans les deux cas, à l'arrière-plan, la fermière délaissée observe le fermier serrant une petite poule dont la courbure du bec est similaire à celle du nez de son propriétaire. Une ressemblance physique est établie entre le fermier et sa poule. Amoureux de sa poule, le vieux semble s'être approprié non seulement une caractéristique physique de la volaille mais surtout la caractéristique luxurieuse attribuée au coq. Il tâte sa poule pour savoir si elle pondra d'ici peu.

Figure 8: Harmen Jansz Müller, Vieux paysan avec sa poule ou le "Tâte-poule ", 1595, burin, $170 \mathrm{~mm}$ de diamètre, Berlin, coll. privée

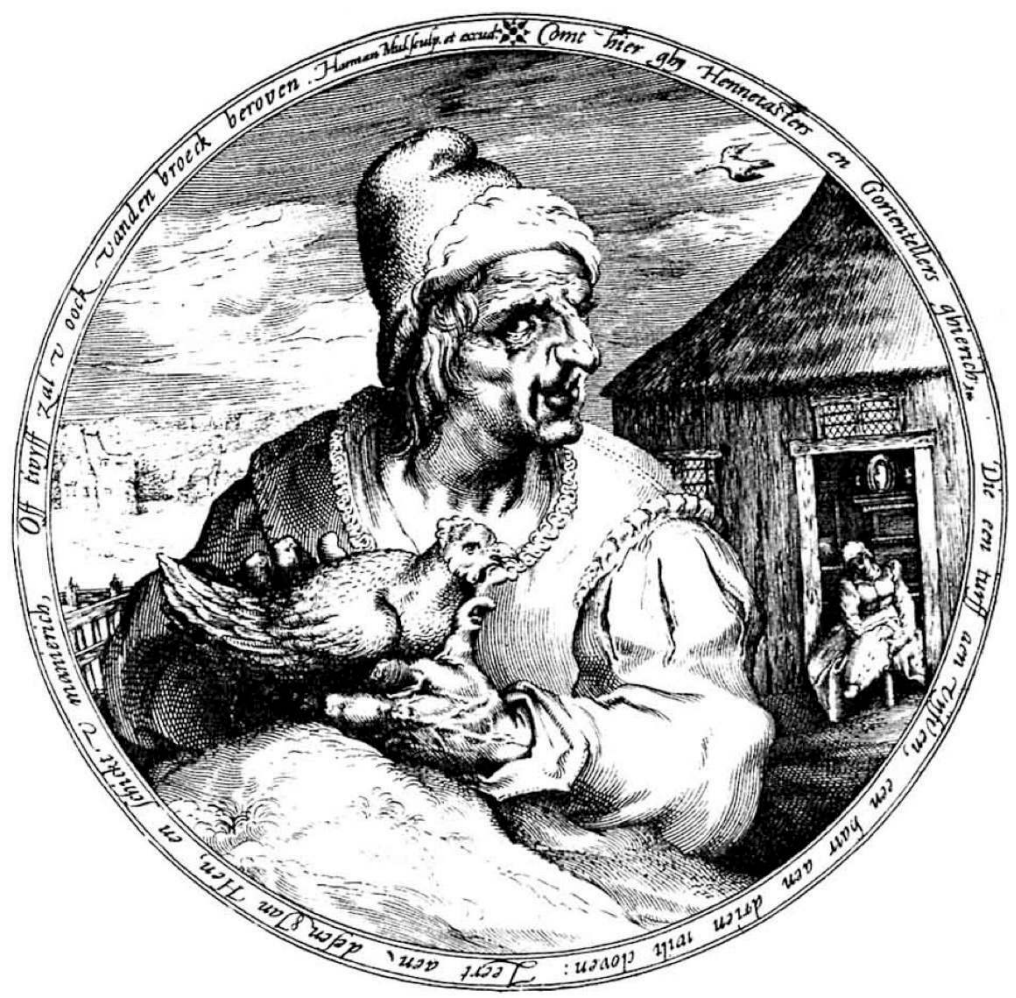


Figure 9 : Hans Liefrinck, Vieux paysan avec sa poule, xvle siècle, xylographie, $400 \times 350 \mathrm{~mm}$, Paris, coll. privée

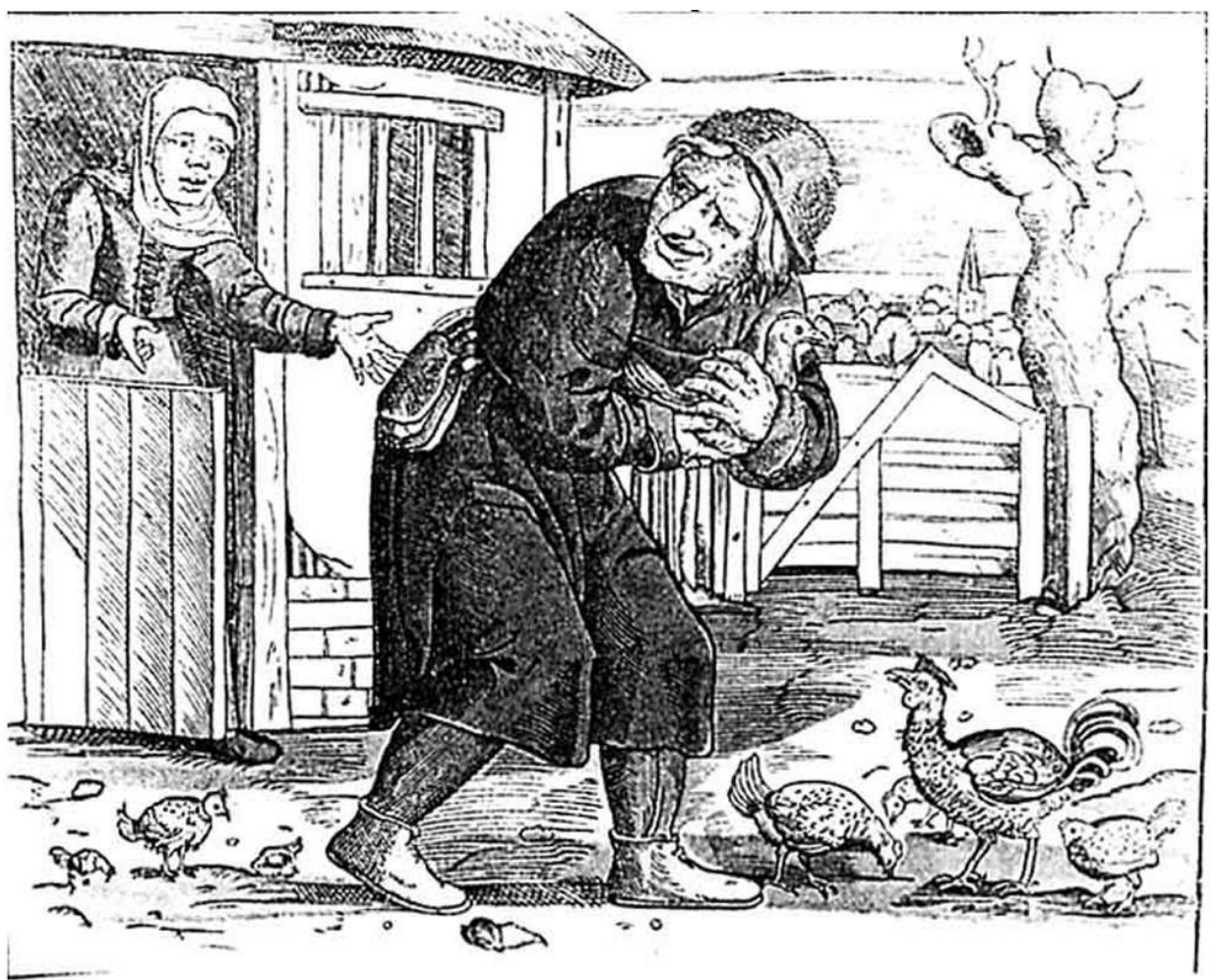

Dans sa composition, Bartolomeo Passerotti reprend le motif du vieux paysan avec sa poule mais en l'inversant ; il y ajoute des échos propres à la culture italienne. Le caractère luxurieux de la viande des volatiles en est un. Nous retrouvons une trace de cette connotation de la viande d'oiseaux dans un des sermons de Bernardin de Sienne, daté de 1427. En expliquant que les veuves et les vieilles femmes appartenaient à la catégorie des individus la moins à l'abri des risques d'échauffements en ingérant une nourriture mal adaptée à leur état, Bernardin disait : «Ne mangez pas de viande d'oiseaux et vivez votre vie d'une façon réglée » (Grieco 1987 : 124). Or, dans l'image, la vieille femme serre dans ses bras un gros coq et l'embrasse. L'aspect risible du désir sexuel est accentué par l'âge avancé de la femme et par l'objet de son désir, l'animal le plus libidineux de la bassecour. Le coq devient attribut de la vieille femme puisque, à son contact, elle a assimilé son caractère libidineux et luxurieux.

Selon les traités diététiques de l'époque, le choix des aliments ingérés est fondamental puisque l'ingestion d'un aliment entraîne l'incorporation des caractéristiques de cet aliment chez la personne qui le mange. Le phénomène est si puissant qu'il peut changer la nature ou le tempérament du mangeur. Dans le cas des personnages et des animaux - qui sont des aliments - montrés sur les toiles de Campi et de Passerotti, l'ingestion n'est pas représentée; c'est la proximité physique homme/animal qui permet le transfert des caractéristiques animales chez l'homme et, inversement, la projection de certains traits humains chez les animaux.

Devant les Volaillers (Figure 1) de Vincenzo Campi, nous ne sommes pas seulement en présence d'une vendeuse et d'un garçon volailler mais aussi devant la figure dévalorisée et blasphématoire d'une Vierge à l'Enfant et celle d'un jeune obsédé sexuel. Devant les Volaillères (Figure 7) de Bartolomeo Passerotti, nous ne sommes pas seulement en 
présence de vendeuses au marché mais aussi devant une entremetteuse et une prostituée. Au-delà de ces seconds niveaux de lecture, un troisième s'y ajoute : celui qui fait de ces tableaux des images de la Luxure. Attributs et symboles, le coq d'un côté, le canard gavé, de l'autre, évoquent, par leur nature, la dinde, par sa forme, des associations d'idées spontanées à la fois avec quelque chose d'absent - l'acte sexuel - et d'abstrait - la luxure. Dans l'image, il suffit d'un rien, d'un regard, d'une posture, d'un détail pour que les doubles sens cachés se révèlent au regard du spectateur. Figures ambiguës par excellence, volailles et volaillères participent de l'équivoque des tableaux de genre des deux artistes italiens.

En investissant leurs tableaux de divers sens, Vincenzo Campi et Bartolomeo Passerotti s'inscrivent dans une tradition où le plaisir du déchiffrement s'associe à des images mentales propres à la Renaissance. La femme, marchande de volailles, dont l'activité essentielle à la ferme est l'élevage des petits oiseaux et l'entretien de la basse-cour, est ici la figure archétypale du monde rural et de ses débordements sociaux et comportementaux. Dévergondée lorsqu'elle est jeune ou libidineuse lorsqu'elle est vieille, elle est souvent associée à des stéréotypes alimentaires comme ici en peinture tout autant que dans un ensemble de textes littéraires et théatraux qui font la spécificité de la culture nord-italienne de la fin du XVI $\mathrm{e}^{\mathrm{e}}$ siècle.

Face à l'utilité de l'image pour l'étude ethno-anthropologique, se pose la question de l'authenticité de la représentation du passé par les œuvres d'art. Les divers niveaux de lecture qu'il est possible de proposer des tableaux de Vincenzo Campi et Bartolomeo Passerotti attestent qu'il faut aussi s'interroger sur les critères qui déterminent la fiabilité de l'image comme document historique. Alors que, au XIX ${ }^{e}$ siècle, on commença à considérer le fait que, concernant les images, «la production courante, moyenne ou de second ordre permettait de comprendre bien plus sûrement la mentalité d'une époque " car «la beauté même des œuvres d'art ne pouvait manquer d'en faire un guide trompeur » (Haskell $1995:$ 484), aujourd'hui, la qualité esthétique n'est plus considérée $a$ priori comme discriminante et les images sont exploitées pour leur valeur descriptive, leur rapport direct au modèle et leur principe de «témoignage visuel » (Burke 2001: 81). Si désormais la beauté ne s'oppose pas au critère de l'utile, l'invention - autre grand principe de la conception de l'art du Cinquecento - doit être prise en considération dans l'analyse des images. Qu'elle soit illustrative ou artistique, l'image possède un « langage » propre que les historiens de l'art tentent de pénétrer par l'analyse des objectifs, des conventions, styles et techniques de chaque représentation. L'image a non seulement le pouvoir de «faire voir ", mais elle est également porteuse de sens, de doubles sens même, et créatrice d'imaginaire.

\section{BIBLIOGRAPHIE}

Alberti L.B. (1435) 1992 - De pictura. Paris, Macula, 269 p.

Baldi M.-L. \& Canziani G. 2002 - Gerolamo Cardano. Un enciclopedista del Rinascimento. Milan, Biblioteca di via Senato, $63 \mathrm{p}$. 
Boudier V. 2010 - La cuisine du peintre. Rennes, PUR, 372 p.

Bougoux C. 1992 - Petite grammaire de l'obscène, églises du duché d'Aquitaine : XI ${ }^{e} / \mathrm{XII}^{e}$ s. Bordeaux, Bellus éditions, $233 \mathrm{p}$.

Burke P. 2001 - Eyewitnessing. The Uses of Images as Historical Evidence. London, Reaktion books, $224 \mathrm{p}$.

De Logu G. 1962 - Natura Morta italiana. Bergame, Instuto Italiano d'Arti Grafiche, 202 p.

Doni A.F. (1552) 1993 - Novella della gentildonna. In : I Marmi, Vigenia, Francisco Marcolin, 93 p.

Estienne C. \& Liebault J. (1550) 1671 - L'agriculture et maison rustique. Rouen, Veuve Guillaume Machuel, $339 \mathrm{p}$.

Gallo A. (1569) 1571 - Secrets de la vraye agriculture, et honestes plaisirs qu'on reçoit en la mesnagerie des champs, divisés en vingt journées par dialogues. Paris, N. Chesneau, 374 p.

Gallo A. 1569 - Le Vinti giornate dell'agricoltura et de'piaceri della villa. Venise, G. Percaccino, 448 p.

Gauricus P. (1504) 1969 - De sculptura. Genève-Paris, Librairie Droz, 293 p.

Grieco A.J. 1987 - Classes sociales, nourriture et imaginaire alimentaire en Italie, XIV ${ }^{e}-\mathrm{XV}^{e}$ siècles, thèse de doctorat. Paris, EHESS, n.p.

Haskell F. (1993) 1995 - L'historien et les images. Paris, Gallimard, 781 p.

Kempf G., Mazzacurati G. \& Motte-Gillet A. 1993 - Conteurs italiens de la Renaissance. Paris, Gallimard, 1926 p. (Bibliothèque de la Pléiade).

Lomazzo G.P. (1584) 1971 - Trattato dell'arte de la Pittura. In : Scritti d'arte del Cinquecento. Milan, Riccardo Ricciardi editore, 2 vol., 2398 p.

Paliaga F. \& De Klerck B. 1997 - Vincenzo Campi, Milan, Edizioni dei Soncino, 297 p.

Patrone A.M.N. (1981) 1989 - Il cibo del ricco ed il cibo del povero. Turin, Centro studi piemontesi, $562 \mathrm{p}$.

Pisanelli B. (1584) 1620 - Traicté de la nature des viandes, et du boire, avec leurs vertus, vices, remèdes, et histoires naturelles, utiles \& delectables à quiquonque desire vivre en santé. Saint-Omer, C. Boscart, $225 \mathrm{p}$.

Testa L. 1995 - La collezione di quadri di Ciriaco Mattei. In : Caravaggio e la collezione Mattei, catalogue d'exposition, Rome, 4 avril-30 mai 1995. Milan, Electa : 29-38.

Wind B. 1975 - Genre as Season: Dosso, Campi, Caravaggio. Arte Lombarda XX (42-43) : 70-73.

\section{NOTES}

1. J'emploie le terme de volaillère dans le sens de vendeuse de volailles. Les tableaux italiens présentés dans ce texte étant intitulés, dans les inventaires, Pollivendoli ou Pollivendole, j'ai pris le parti d'utiliser le mot Volaillère au lieu de l'expression Marchande de volailles.

2. Le terme "genre » en histoire de l'art soulève une difficulté. Il définit la nature d'un sujet de représentation: le genre du portrait, le genre de la nature morte ou de la scène de genre. La définition de l'expression "peinture de genre " pose encore problème. Jusqu'au XVIII ${ }^{\mathrm{e}}$ siècle, c'est une catégorie regroupant tout ce qui n'est pas peinture d'histoire tandis que, à partir du XIX e siècle, elle illustre des scènes de la vie quotidienne. 
3. Très souvent des fermières ; «Quant au gouvernement des poules, qui est le principal état de la Fermière, elle doit être soigneuse [...]» (Estienne \& Liébault $1671: 66$ ).

4. CEuvre (vers 1580, huile sur toile, 77 x $89 \mathrm{~cm}$ ) conservée actuellement au Musée des Beaux-Arts de Lyon.

5. CEuvre (vers 1580, huile sur toile, 145 x $210 \mathrm{~cm}$ ) conservée actuellement à la Pinacothèque de Brera, Milan.

6. CEuvre (vers 1580, huile sur toile, $145 \times 215 \mathrm{~cm}$ ) conservée actuellement à la Pinacothèque de Brera, Milan.

7. CEuvre (vers 1580, huile sur toile, $145 \times 216 \mathrm{~cm}$ ) conservée actuellement à la Préfecture de Crémone.

8. CEuvre (vers 1580 , huile sur toile, $145 \times 220 \mathrm{~cm}$ ) conservée actuellement à la Pinacothèque de Brera, Milan.

9. Des Pietre di paragone comme les décrit l'acte notarié de 1611.

10. CEuvre (1580, huile sur toile, 135 x $220 \mathrm{~cm}$ ) conservée actuellement au château de Kirchheim, Bavière.

11. Euvre (vers 1580, huile sur toile, $135 \times 220 \mathrm{~cm}$ ) conservée actuellement au château de Kirchheim, Bavière.

12. Cuvres (1578, huile sur toile, $135 \times 220 \mathrm{~cm}$; 1581, huile sur toile, $135 \times 220 \mathrm{~cm}$ ) conservées actuellement au château de Kirchheim, Bavière.

13. Les penseurs des $\mathrm{XV}^{\mathrm{e}}$ et $\mathrm{XVI}^{\mathrm{e}}$ siècles apparentent la notion de varietas (variété) à celle de copia (abondance). Dans la lignée des rhéteurs de l'Antiquité, Leon Battista Alberti, dans son De pictura de 1435, explique comment l'abondance du peintre doit être soumise au principe sélectif de variété.

14. Alors que le canard, la dinde, les coqs, les poules, les chapons sont des animaux domestiques, les bécasses, le faisan et la faisane ainsi que les perdrix grises et les étourneaux (sansonnets) sont des animaux sauvages.

15. Publié en 1550, l'ouvrage connaît dès 1551 des rééditions à Paris et à Lyon. D'autres suivent en 1554 et 1559.

16. Publié en 1557, l'ouvrage est réédité l'année suivante et connaît dès 1559 une traduction allemande sous le titre Offenbarung der Natur. D'autres rééditions suivent en 1580 et 1581.

17. Les Poissonniers, et leurs espèces animales qui peuplent les mers, forment pendant aux Volaillers qui regroupent les espèces volatiles.

18. Nous songeons aux liens plastiques entre la Poissonnière et les Poissonniers, de Kirchheim.

19. Le thème de la virilité vigoureuse des paysans est déjà traité chez Boccace, celui de la performance amoureuse est abordé chez Bandello.

20. À moins qu'il ne gonfle ses joues insistant sur le rôle du pneuma, à la fois air et sperme selon la théorie hippocratique.

21. Au premier plan, le récipient étamé contenant du grain et l'entonnoir à gaver éclairent le geste fait par le garçon. Un geste incompris, dès le xix ${ }^{\mathrm{e}}$ siècle, comme l'atteste la notice du catalogue de vente de la collection Francesco Molinari de 1885, décrivant cette œuvre comme «Una donna tiene in mano un tacchino spennato. Un ragazzo torce il collo ad un'oca » cité dans la notice 26 de l'ouvrage de Paliaga et de De Klerck (Paliaga \& De Klerck 1997). Dans la continuité de cette lecture, les mêmes auteurs y voient « un ragazzo, con le guance gonfie, sta soffocando un'oca » (Ibid.).

22. Traduction personnelle de la description des tableaux issue de l'inventaire de 1614 .

23. Le lever de jambe est un type majeur d'exhibitionnisme féminin, mais aussi masculin comme l'atteste le Tireur d'épine - Spinario - à l'origine, un bronze grec $\mathrm{du} \mathrm{V}^{\mathrm{e}}$ siècle avant J.-C. où la jambe levée est soit un geste délibérément obscène, soit un prétexte à exposer les parties génitales du garçon à la vue de tous. 
24. Citons, entre autres, Henry Corneille Agrippa, Traité de l'excellence de la femme, traduction du latin au français de Lois Vivant, Paris, J. Poupy, 1578.

25. L'animal peint est l'archétype du beau et bon coq : « [...] il ne faut point qu'il soit blanc ni gris : mais ou roux, ou noir, le corps bien entassé, la crête bien droite, vermeille, épaisse, non crénelée, d'entelée, ni chiquetée, le col relevé \& haut, [...] les ouies grandes \& fort blanches, le bec court, gros \& crochu, [...] le plumage du col bien long, doré \& changeant, les jambes bien ecaillées, grosses \& courtes, l'ongle court \& ferme, l'ergot roide \& pointu » (Estienne \& Liebault $1671: 67)$.

\section{RÉSUMÉS}

Les œuvres peintes ne sont nullement aisées à déchiffrer et les scènes de vente de volailles proposées par les artistes nord-italiens Vincenzo Campi et Bartolomeo Passerotti sont loin d'être des instantanés de la vie populaire du XVI ${ }^{\mathrm{e}}$ siècle italien. L'image artistique possède un langage propre que les historiens de l'art tentent de pénétrer par l'analyse des objectifs, des conventions, styles et techniques de chaque représentation. Cette analyse plastique doit être croisée avec l'étude de textes contemporains de tous types (littéraire, artistique, culinaire) et cela pour permettre une ouverture de l'interprétation vers un arrière-plan social et culturel. C'est ce croisement dans l'analyse qui nous permet d'avancer que, dans les tableaux, le motif de la volaille et celui de la volaillère possèdent une place et un traitement tout à fait originaux. Ils semblent incarner les modalités de regard du spectateur dans l'économie des tableaux de genre des deux artistes italiens du Cinquecento.

Painted works are not easy to decipher and scenes of poultry sale by North Italian artists Vincenzo Campi and Bartolomeo Passerotti are not snapshots of every day popular life of the Sixteenth Century, in Italy. Artistic image has a language of its own the Art historians attempt to decipher by analyzing the objectives, conventions, styles, and techniques of each work. This plastic analysis must be crossed with the study of literary, artistic and culinary texts of those times, and this in order for the analysis to encompass a social and cultural background. It's this crossing in the analysis that allows us to suggest that the topic of the poultry and poultries's seller have place and pictorial treatment quite original in paintings. They seem to embody the modalities of the viewer's gaze in the pictorial organization of genre scenes by these two North Italian artists of the Sixteenth Century.

\section{INDEX}

Mots-clés : volaille, volaillère, Campi, Passerotti, peinture, Cinquecento

Keywords : poultry, poultries's sellers, Campi, Passerotti, picture

\section{AUTEUR}

\section{VALÉRIE BOUDIER}

valerie.boudier@univ.lille3.fr 